\section{A retrospective comparative study of the efficacy and safety of two regimens of diphenylcy- clopropenone in the treatment of recalcitrant alopecia areata}

\author{
Tueboon Sriphojanart, Saranya \\ Khunkhet, Poonkiat Suchonwanit \\ Division of Dermatology, Faculty \\ of Medicine, Ramathibodi Hospital, \\ Mahidol University, Bangkok, Thailand
}

\begin{abstract}
Diphenylcyclopropenone (DPCP) is an effective topical immunotherapy for recalcitrant alopecia areata (AA), which sometimes requires prolonged treatment. We developed a new treatment protocol to shorten the duration of therapy. This study aimed to compare the efficacy and safety of the new treatment protocol with the standard treatment protocol in the treatment of recalcitrant AA. We conducted a 6-year retrospective comparative study of patients with AA who received one of the DPCP treatment protocols at our institute. Patients' information was collected and subsequent statistically analyzed. Thirtynine patients (16 in the new treatment group and 23 in the standard treatment group) were included. There were no statistically significant differences in area of hair regrowth. Mean duration to initial hair regrowth and mean duration to significant hair regrowth in the new treatment group were significantly shorter than in the standard treatment group $(\mathrm{P}=0.002$ and 0.01 , respectively). Adverse effects were slightly higher in the new treatment group. The present study reveals the effectiveness and safety of the new treatment protocol, which shortens the duration of DPCP treatment and could represent an alternative regimen.
\end{abstract}

\section{Introduction}

Alopecia areata (AA) is an autoimmune disease with a genetic basis presenting with non-scarring hair loss both on the scalp and non-scalp areas. The prevalence of AA is approximately $1-2 \%$ of the general population. ${ }^{1}$ The disease has no sexual and age prevalence. Clinically, patients manifest with well-defined, asymptomatic, non-scarring areas of hair loss with characteristic exclamation mark hairs. The prognosis of the disease is quite variable. Spontaneous improvement within 1 year was reported in $34-50 \%$ of patients. However, in $14-25 \%$, AA can progress to total scalp hair loss (alopecia totalis; AT) or even entire body hair loss (alopecia universalis; AU), which shows spontaneous improvement in less than $10 \%$ of cases. ${ }^{2}$ Even though AA is not a life threatening disease, it can greatly affect patients' psychosocial status and quality of life. Compared with the general population, psychological disorders, such as anxiety, depression, and phobias, are more common in patients with AA. ${ }^{3,4}$

The pathogenesis of AA remains to be determined. Currently, a widely accepted theory is the autoimmune etiology. Specific T-cell lymphocytes, autoantibodies against anagen follicles, and various cytokines such as interferon- $\gamma$, interleukins, and tumor necrosis factor- $\alpha$ have been found to play a major role in AA. ${ }^{5}$ In addition, the immune privilege theory has been recently introduced and suggested to play a role in the pathogenesis. ${ }^{6}$ Therefore, the principle of treatment is to inhibit all the possible etiological pathways. There is currently no curative treatment for AA. Treatment modalities include corticosteroids, anthralin, cyclosporine, biologic therapy, topical immunotherapy, photochemotherapy, and 308-nm excimer laser, among other. $^{7}$ However, treatment is still very difficult, particularly in patients with chronic and extensive AA.

Currently, topical immunotherapy using diphenylcyclopropenone (DPCP) is one interesting treatment option for recalcitrant case. This treatment was first introduced by Happle et al. in 1983. ${ }^{8}$ The principle of treatment is induction and repeated elicitation of an allergic contact dermatitis by application of contact allergen on affected areas. Its mechanism of action is not fully understood, but antigenic competition from induction of contact dermatitis on the scalp is believed to be a possible mechanism. ${ }^{9}$

For almost 10 years, we have been performing DPCP treatment regularly in patients with AA who do not respond to other treatments within the first 6 months. The first step of treatment is sensitization of the patient to DPCP. After this is successfully completed, the lowest concentration of DPCP is initially applied to the affected area on the patient's scalp. Then, the concentration is gradually increased every week until the optimal reaction occurs. We observed that some patients took several weeks or even months to reach the optimal reaction, which could extend the overall treatment duration.

We developed a new treatment protocol of topical immunotherapy for recalcitrant AA and have been using it in some patients
Correspondence: Poonkiat Suchonwanit, Division of Dermatology, Ramathibodi Hospital, 270 Rama VI Road, Rajthevi, Bangkok, Thailand 10400 .

Tel.: +662.201.1141 - Fax: +662.201.1211. E-mail:poonkiat@hotmail.com

Key words: Alopecia; alopecia areata; diphencyprone; diphenylcyclopropenone; topical immunotherapy.

Contributions: TS, SK, PS, study conception and design; TS, SK, acquisition of data; TS, SK, PS, analysis and interpretation of data; TS, PS, drafting of manuscript; PS, critical revision.

Conflict of interest: The authors declare no conflict of interest.

Received for publication: 7 September 2017. Accepted for publication: 6 November 2017.

This work is licensed under a Creative Commons Attribution-NonCommercial 4.0 International License (CC BY-NC 4.0).

(C) Copyright T. Sriphojanart et al., 2017 Licensee PAGEPress, Italy

Dermatology Reports 2017; 9:7399

doi:10.4081/dr.2017.7399

since 2011. The purpose of developing the new protocol was to minimize the duration of DPCP treatment. After sensitization, multiple concentrations of DPCP were applied to the scalp in individual small areas at the same time. Then, the optimal concentration that could cause mild contact dermatitis was selected to be the first concentration applied to the patient's scalp. We believe that our new treatment protocol could reduce the treatment duration and also reduce the cumulative treatment cost compared to the standard protocol. Therefore, to prove our hypothesis, we conducted a retrospective comparative study of patients with AA who received DPCP treatment at our institute. The objective of the study was to determine whether the new treatment protocol is superior to the standard protocol in the treatment of recalcitrant AA and to compare adverse events between the two treatment protocols.

\section{Materials and Methods}

\section{Study design}

This is a retrospective comparative study conducted at Ramathibodi Hospital, Mahidol University. Patients' information from 2011 to 2016 was collected from medical records. The study was reviewed and 
approved by the Mahidol University Institutional Review Board for Ethics in Human Research (protocol number 06-5952).

\section{Patients}

Patients with AA who received DPCP treatment using either the standard treatment protocol or the new treatment protocol in the outpatient dermatology clinic were included in the study. The diagnosis of AA in each patient was based on clinical and/or histological examination. The inclusion criteria were age greater than 18 years, unfavorable treatment outcomes with any other topical and systemic treatments for at least 6 months, scalp hair loss greater than $50 \%$, and regular followed up in our clinic at least 6 months after discontinuation of DPCP treatment. The exclusion criteria were incomplete medical records and unsuccessful sensitization to DPCP. Demographic data including the age of onset, duration of disease, medical history, family history of AA, type of AA, severity of disease measured using the Severity of Alopecia Tool (SALT) score, ${ }^{10}$ and nail abnormalities were collected for further analysis.

\section{Sensitization to diphenylcyclo- propenone}

DPCP solution was prepared by dissolving DPCP powder (Fluka, SigmaAldrich Corp, St. Louis, MO, USA) in acetone at serial dilutions of $0.0001 \%$, $0.0005 \%, 0.001 \%, 0.005 \%, 0.01 \%, 0.02 \%$, $0.05 \%, 0.1 \%, 0.2 \%, 0.5 \%, 1.0 \%$, and $2.0 \%$ and storing the dilution in dark glass vials to prevent degradation from ultraviolet light. At the first visit, the patient was sensitized by application of $2 \%$ DPCP at the inner aspect of the upper arm over a $4 \times 4 \mathrm{~cm}^{2}$ area. DPCP was left on the sensitized area for 24 hours then washed off. After $72 \mathrm{~h}$, patient was evaluated for an eczematous reaction to detect whether sensitization to DPCP had occurred.

\section{Treatment with standard regimen}

Two weeks after sensitization, DPCP treatment starting with the $0.0001 \%$ concentration was applied at the affected scalp area every week. First, DPCP was left on the scalp for $24 \mathrm{~h}$ and then washed off with gentle shampoo. Each week, concentration of DPCP was titrated upward to reach a tolerable eczematous reaction, which was mild pruritus and mild erythema lasting not more than $48 \mathrm{~h}$. The concentration that produced optimal eczematous reaction was maintained at subsequent treatments. If the concentration of DPCP used on the scalp revealed negative or below the optimal reaction, then the concentration of $\mathrm{DPCP}$ would be titrated upward. The concentration may be titrated upward or downward to maintain the optimal eczematous reaction.

\section{Treatment with new treatment regimen}

Two weeks after sensitization, the scalp was mapped for applying DPCP. Then, six consecutive concentrations of DPCP (0.0001\%, 0.001\%, 0.01\%, 0.05\%, 0.1\%, and $0.5 \%$ ) were applied on the scalp over $3 \times 3 \mathrm{~cm}^{2}$ areas separated by a distance of 4 $\mathrm{cm}$. DPCP was left on the scalp for $24 \mathrm{~h}$ and then washed off with gentle shampoo. One week later, the concentration that created a mild eczematous reaction was chosen to be the first applied. The starting concentration was applied at the affected scalp area and left for $24 \mathrm{~h}$ and then washed off. The concentration of DPCP was adjusted every week to maintain a tolerable eczematous reaction.

\section{Clinical assessment}

The patients were followed up weekly for evaluation of clinical responses and side effects. Treatment was withdrawn if there were no signs of hair regrowth at 6 months. If there was any clinical response, treatment was continued until complete hair regrowth. For patients with incomplete hair regrowth at 1 year after treatment, percentage of hair regrowth at 1 year was recorded as their treatment outcome. All patients were followed up monthly for 6 months after discontinuation of treatment.

The clinical assessment was divided into efficacy and safety. Efficacy assessment included the treatment response reported as percentages of area of hair regrowth, change in SALT score from baseline, and duration of clinical responses (initial response and significant response). The initial response was defined as appearance of any new regrowth hair within treated sites and significant response was defined as greater than $75 \%$ hair regrowth. Failure of treatment was considered when hair regrowth was not observed after the first 6 months of treatment. Relapse was defined as more than $25 \%$ hair loss after complete hair regrowth. Safety information was collected from the adverse effects recorded.

\section{Statistical analysis}

Statistical analysis was performed using the SPSS statistics version 18.0 (SPSS Inc., Chicago, IL, USA). Chi-square test, Fisher exact test, t-test, and Wilcoxon rank-sum test were performed to compare demographic data, efficacy, and adverse effects. The results were considered statistically significant at $\mathrm{P}<0.05$.

\section{Results}

From a total of 65 patients with AA who received DPCP treatment during the last 5 years, 39 patients were eligible and were included in the study. Sixteen patients were treated with the new treatment regimen and 23 patients were treated with the standard regimen. Epidemiological data and baseline clinical characteristics of all patients are compared and summarized in Table 1. The groups were comparable in terms of age, gender, duration of disease, underlying disease, scalp area involvement, and nail involvement.

Table 2 demonstrates the comparison of treatment outcomes between the 2 treatment groups. Eight patients in the new treatment group and 12 patients in the standard group had $>75 \%$ area of hair regrowth. Seven patients in each group had complete hair regrowth. Failure of treatment was reported in 2 and 3 patients in the new and standard treatment group, respectively. Regarding area of hair regrowth, there were no statistically significant differences between the two groups. For treatment duration, mean duration to initial hair regrowth in the new treatment group was significantly shorter than that in the standard treatment group $(10.5 \pm 2.6$ weeks and $14.2 \pm 3.9$ weeks, respectively; $\mathrm{P}=0.002$ ). Moreover, mean duration to $>75 \%$ hair regrowth was $24.3 \pm 4.2$ weeks in the new treatment group and $29.2 \pm 6.5$ weeks in the standard treatment group, representing a statistically significant difference $(\mathrm{P}=0.01)$. Regarding relapse of AA, there was no significant difference in both number of patients and median duration to relapse between the 2 groups ( $\mathrm{P}=0.81$ and 0.62 , respectively). Adverse effects were slightly higher in the new treatment group, without statistical significance.

\section{Discussion}

Topical immunotherapy using DPCP has been proven effective in the treatment of patients with recalcitrant AA. This method is recommended as first-line therapy in patients with AA who have more than $50 \%$ scalp area involvement. ${ }^{11}$ Its efficacy, in terms of acceptable regrowth, has been reported in previous studies with variable response. A systematic review reported that the average response rate of DPCP treatment in 26 studies was $53.75 \%{ }^{12}$ Our study reported a significant response rate of $50 \%$ in the new treatment group and $52.1 \%$ in the standard treatment group, which are comparable with the average response rate. 
Previous studies reported response rates of DPCP ranging from 5 to $85 \%^{8,13-20}$ The variability in clinical response rate between studies may be attributed to different study designs, treatment protocols, baseline disease severity, therapeutic response grading systems, and follow-up periods. To minimize this problem in future studies, clinical trials using a standardized method in clinical assessment is recommended.

Regarding percentage of hair regrowth, the present study found similar clinical response rates between the 2 groups. The data confirms that the efficacy of the new treatment protocol was not inferior in comparison with the standard treatment protocol. In addition, this retrospective study reveals the advantage of the multiple DPCP treatment protocol in shortening the duration of treatment. Our study showed that mean duration to initial response and mean duration to $>75 \%$ hair regrowth in the new treatment group were significantly shorter than in the standard treatment group, which was approximately 5 weeks. Our results are comparable with a previous study using multiple concentrations of DPCP in patients with AT or AU, which revealed a benefit of shortened treatment duration..$^{21}$

When comparing the treatment duration using data from the standard treatment group, the average duration to significant hair regrowth was comparable with previous studies. Chiang et al. ${ }^{22}$ and El Khoury et $a l .23$ reported durations to significant hair regrowth of 28 and 31.74 weeks, respectively. Therefore, the new treatment protocol could shorten the response time, which normally takes several months to reach a significant clinical response, and could be considered an alternative regimen to shorten the therapeutic period. Application of multiple concentrations of DPCP at the first treatment assisted the physician in identifying the optimal concentration in a shorter period. Reduction of the overall treatment duration could reduce the cost of treatment and other expenses such as transportation, accommodation, and income loss while undergoing DPCP treatment.

The relapse rates in each treatment group in the present study were less than that reported in previous studies, which range between 45.1 and $70 \% .^{19,20,24-26}$ The difference in the relapse rate may be due to different definitions of relapse and the duration of follow-up. Although the relapse rate of topical immunotherapy with DPCP is quite high, it is considered to be the most successful treatment for severe types of AA. ${ }^{27}$

Common adverse effects of DPCP treatment described in the literature are severe eczematous reaction, wide spread eczema, urticarial reaction, severe dermographism, and cervical lymphadenopathy. ${ }^{28-30}$ The present study shows that adverse effects were slightly higher in the new treatment protocol group, without statistical significance. Using a higher concentration during the early treatment period could increase the possibility of severe reactions. Fortunately, the adverse effects were tolerable and rapidly disappeared within a few days after providing symptomatic treatment. None of the patient dropped out of the treatment.

Limitations of this study are the retro-

spective design and small number of patients. Therefore, we were unable to assess patients' history and clinical evaluation completely. The small sample size may account for the lack of statistically significant results. Prospective clinical trials with a large number of patients could overcome this limitation.

\section{Conclusions}

Topical immunotherapy is an effective treatment option for patients with chronic

Table 1. Demographics and clinical characteristics of the patients in the 2 treatment groups.

\begin{tabular}{lccc}
\hline Variables & Standard regimen & New regimen & P value \\
Number & 23 & 16 & N/A \\
Mean age (year) & $35.5 \pm 5.2$ & $32.9 \pm 4.6$ & 0.11 \\
\hline Sex (male:female) & $9: 14$ & $6: 10$ & 0.91 \\
Mean age at onset (year) & $26.9 \pm 5.6$ & $23.5 \pm 5.8$ & 0.07 \\
\hline Mean duration of disease (week) & $56.9 \pm 10.2$ & $61.9 \pm 12.6$ & 0.17 \\
Underlying diseases, $n(\%)$ & & & \\
Atopic disease & $3(13)$ & $1(6.2)$ & 0.49 \\
Autoimmune thyroid & $1(4.3)$ & $1(6.2)$ & 0.79 \\
\hline Family history of AA, $n(\%)$ & $2(8.6)$ & $3(18.8)$ & 0.35 \\
Nail involvement, $n$ (\%) & $8(34.8)$ & $9(56.2)$ & 0.18 \\
\hline Type of alopecia areata, $n(\%)$ & & & \\
Multiple patches & $9(39.2)$ & $6(37.4)$ & 0.81 \\
Alopecia totalis & $8(34.8)$ & $7(43.7)$ & \\
Alopecia universalis & $6(26)$ & $3(18.9)$ & \\
Baseline area involvement, $n(\%)$ & $5(21.7)$ & $3(18.9)$ & 0.95 \\
51-75\% & $5(21.7)$ & $4(25)$ & \\
$76-99 \%$ & $13(56.6)$ & $9(56.1)$ & \\
100\% & & & \\
\hline
\end{tabular}

AA, alopecia arata.

Table 2. Response to treatments and adverse effects observed in patients in the 2 treatment groups.

\begin{tabular}{lccc}
\hline Variables & Standard regimen & New regimen & P value \\
Area of hair regrowth, $\mathrm{n}(\%)$ & & & \\
$\quad$ no hair regrowth & $3(13)$ & $2(12.5)$ & 0.99 \\
$1-25 \%$ & $2(8.6)$ & $2(12.5)$ & \\
$26-50 \%$ & $2(8.6)$ & $1(6.2)$ & \\
$51-75 \%$ & $4(17.7)$ & $3(18.9)$ & \\
$76-99 \%$ & $5(21.7)$ & $1(6.2)$ & \\
$100 \%$ & $7(30.4)$ & $7(43.7)$ & \\
Mean duration to initial response (week) & $14.2 \pm 3.9$ & $10.5 \pm 2.6$ & $0.002^{*}$ \\
\hline Mean duration to $>75 \%$ hair regrowth (week) & $29.2 \pm 6.5$ & $24.3 \pm 4.2$ & $0.01 *$ \\
Relapse, $n$ (\%) & $5(21.7)$ & $4(25)$ & 0.81 \\
\hline Median duration to relapse (week) & $14(8-23)$ & $11(6-18)$ & 0.62 \\
Adverse effects, $n$ (\%) & & & \\
Blister formation & $1(4.3)$ & $1(6.2)$ & 0.9 \\
Widespread eczema & $3(13)$ & $6(37.4)$ & \\
Dyspigmentation & $1(4.3)$ & $2(12.5)$ & \\
Lymphadenopathy & $7(30)$ & $8(50)$ & \\
\hline
\end{tabular}

*Statistically significant; ${ }^{*}$ data collected from patients with $>75 \%$ hair regrowth. 
and extensive AA. The present study shows that the new treatment protocol is effective and safe, and could shorten the duration of DPCP treatment. Our new treatment protocol could be an alternative regimen for the treatment of AA.

\section{References}

1. Willemsen R, Vanderlinden J, Roseeuw D, Haentjens P. Increased history of childhood and lifetime traumatic events among adults with alopecia areata. J Am Acad Dermatol 2009;60:388-93.

2. Mcdonagh AJ, Messenger AG. The pathogenesis of alopecia areata. Dermatol Clin 1996;14:661-70.

3. Rafique R, Hunt N. Experiences and coping behaviours of adolescents in Pakistan with alopecia areata: an interpretative phenomenological analysis. Int J Qual Stud Health Well-being 2015;10:26039.

4. Hunt N, Mchale S. The psychological impact of alopecia. BMJ 2005;331:9513.

5. Gregoriou S, Papafragkaki D, Kontochristopoulos G, et al. Cytokines and other mediators in alopecia areata. Mediators Inflamm 2010;2010:928030.

6. Kang H, Wu WY, Lo BK, et al. Hair follicles from alopecia areata patients exhibit alterations in immune privilegeassociated gene expression in advance of hair loss. J Invest Dermatol 2010;130:2677-80.

7. Delamere FM, Sladden MM, Dobbins HM, Leonardi-Bee J. Interventions for alopecia areata. Cochrane Database Syst Rev 2008:Cd004413.

8. Happle R, Hausen BM, WiesnerMenzel L. Diphencyprone in the treatment of alopecia areata. Acta Derm Venereol 1983;63:49-52.

9. Sutherland L, Laschinger M, Syed ZU, Gaspari A. Treatment of alopecia areata with topical sensitizers. Dermatitis 2015;26:26-31.

10. Olsen EA, Hordinsky MK, Price VH, et al. Alopecia areata investigational assessment guidelines-Part II. National Alopecia Areata Foundation. J Am Acad Dermatol 2004;51:440-7.

11. Alkhalifah A, Alsantali A, Wang E, et al. Alopecia areata update: part II. Treatment. J Am Acad Dermatol 2010;62:191-202.

12. Jang YH, Jung HJ, Moon SY, et al. Systematic review and quality analysis of studies on the efficacy of topical diphenylcyclopropenone treatment for alopecia areata. J Am Acad Dermatol 2017;77:170-2.

13. Hull SM, Cunliffe WJ. Successful treatment of alopecia areata using the contact allergen diphencyprone. $\mathrm{Br} \mathrm{J}$ Dermatol 1991;124:212-3.

14. Van Der Steen PH, Van Baar HM, Perret CM, Happle R. Treatment of alopecia areata with diphenylcyclopropenone. J Am Acad Dermatol 1991;24:253-7.

15. Van Der Steen PH, Boezeman JB, Happle R. Topical immunotherapy for alopecia areata: re-evaluation of 139 cases after an additional follow-up period of 19 months. Dermatology 1992;184:198-201.

16. Cotellessa C, Peris K, Caracciolo E, et al. The use of topical diphenylcyclopropenone for the treatment of extensive alopecia areata. J Am Acad Dermatol 2001;44:73-6.

17. Aghaei S. Topical immunotherapy of severe alopecia areata with diphenylcyclopropenone (DPCP): experience in an Iranian population. BMC Dermatol 2005;5:6.

18. Firooz A, Bouzari N, Mojtahed F, et al. Topical immunotherapy with diphencyprone in the treatment of extensive and/or long-lasting alopecia areata. J Eur Acad Dermatol Venereol 2005;19:393-4.

19. Avgerinou G, Gregoriou S, Rigopoulos $\mathrm{D}$, et al. Alopecia areata: topical immunotherapy treatment with diphencyprone. J Eur Acad Dermatol Venereol 2008;22:320-3.

20. Ohlmeier MC, Traupe H, Luger TA, Bohm M. Topical immunotherapy with diphenylcyclopropenone of patients with alopecia areata - a large retrospec- tive study on 142 patients with a selfcontrolled design. J Eur Acad Dermatol Venereol 2012;26:503-7.

21. Thuangtong R, Varothai S, Triwongwaranat D, Rujitharanawong C. Multi-concentration level patch test guided diphenyl cyclopropenone (DPCP) treatment in alopecia totalis or alopecia universalis, J Med Assoc Thai 2017;100:86-92.

22. Chiang KS, Mesinkovska NA, Piliang MP, Bergfeld WF. Clinical Efficacy of Diphenylcyclopropenone in alopecia areata: retrospective data analysis of 50 patients. J Investig Dermatol Symp Proc 2015;17:50-5.

23. El Khoury J, Abd-El-Baki J, Succariah F, et al. Topical immunomodulation with diphenylcyclopropenone for alopecia areata: the Lebanese experience. Int J Dermatol 2013;52:1551-6.

24. Bolduc C, Shapiro J. DPCP for the treatment of alopecia areata. Skin Therapy Lett 2000;5:3-4.

25. Wiseman MC, Shapiro J, Macdonald N, Lui H. Predictive model for immunotherapy of alopecia areata with diphencyprone. Arch Dermatol 2001;137:1063-8.

26. Luk NM, Chiu LS, Lee KC, et al. Efficacy and safety of diphenylcyclopropenone among Chinese patients with steroid resistant and extensive alopecia areata. J Eur Acad Dermatol Venereol 2013;27:e400-5.

27. Hoffmann R, Happle R. Topical immunotherapy in alopecia areata. What, how, and why? Dermatol Clin 1996;14:739-44.

28. Hull SM, Cunliffe WJ. Treatment of alopecia areata with diphenylcyclopropenone. J Am Acad Dermatol 1992;26:276-7.

29. Alam M, Gross EA, Savin RC. Severe urticarial reaction to diphenylcyclopropenone therapy for alopecia areata. J Am Acad Dermatol 1999;40:110-2.

30. Skrebova N, Nameda Y, Takiwaki H, Arase S. Severe dermographism after topical therapy with diphenylcyclopropenone for alopecia universalis. Contact Dermatitis 2000;42:212-5. 